\title{
Évaluation de l'activité antifongique et de la phytotoxicité de Isoberlinia doka craib \& staff
}

\author{
Harouna Ouédraogo, Ingénieur
}

Centre Agricole Polyvalent de Matourkou, Bobo-Dioulasso, Burkina Faso

Lassina Ouattara, PhD

Paulin Ouoba, PhD

Université Nazi BONI / Unité de Formation et de Recherche en sciences et Techniques, Bobo-Dioulasso, Burkina Faso

Schémaeza Bonzi, PhD

Irénée Somda, Professeur

Université Nazi BONI / Institut de Développement Rural,

Bobo-Dioulasso, Burkina Faso

Doi: 10.19044/esj.2018.v14n30p213 URL:http://dx.doi.org/10.19044/esj.2018.v14n30p213

\begin{abstract}
This study has been designed to contribute to search alternative solutions based on plant extracts against phytopatogenic fungi. It aims to test the efficacy of ethanolic extract (70\%) of Isoberlinia doka trunk bark on the mycelial growth of eight phytopagenic fungi species and on maize and cowpea seedlings germination and growth. The results showed that the ethanolic extract inhibits mycelial growth. This extract has showed an interesting efficiency on three fungi such as Colletotrichum dematium, Curvularia lunata and Fusarium verticilloides. Among these fungi, Curvularia lunata was the most sensitive with a rate of inhibition of mycelial growth of $52.35 \%$ after four days incubation at $10 \mathrm{mg} / \mathrm{ml}$. After seven days incubation, the extract was more effective in reducing Fusarium verticilloides mycelial growth with $50,70 \%$ inhibition rate at the same concentration. After the phytotoxicity tests, it was found that this extract had no phytotoxic effect on the germination and growth of maize seedlings while it reduced significantly seed germination and cowpea seedling growth. The ethanolic extract of $I$. doka bark has antifungal properties and did not inhibited maize growth parameters. It could be used in maize seed treatment. Investigations could also be made into the herbicidal properties of some invasive legumes close to cowpea.
\end{abstract}

Keywords: Isoberlinia doka, ethanol extract, inhibition, fungi, phytotoxicity 


\section{Résumé}

La présente étude est une contribution à la recherche de solutions alternatives basées sur des extraits de plantes pour lutter contre les champignons phytopathogènes. L'objectif est de tester l'efficacité de l'extrait éthanolique $(70 \%)$ de l'écorce de tronc de Isoberlinia doka sur la croissance mycélienne de huit espèces de champignons phytopagènes. En outre la toxicité de l'extrait sur la germination des graines et la croissance des plantules de maïs et de niébé a été évaluée. Les résultats ont montré que l'extrait éthanolique inhibe la croissance mycélienne. Cet extrait a inhibé signitifativement la croissance de trois champignons tels que Colletotrichum dematium, Curvularia lunata et Fusarium verticillioides. Parmi ces champignons, $C$. lunata a été le plus sensible avec un taux d'inhibition de 52,35\% après quatre jours d'incubation à $10 \mathrm{mg} / \mathrm{ml}$. Après sept jours d'incubation, l'extrait a été plus efficace et plus élevée sur Fusarium verticillioides avec un taux d'inhibition de $50,70 \%$ à la même concentration. Le test de phytotoxicité n'a pas montré d'effet inhibiteur sur la germination des graines et la croissance des plantules de maïs. Par contre l'extrait a réduit de manière significative la germination des graines et la croissance des plantules de niébé. L'extrait éthanolique de l'écorce de $I$. doka a des propriétés antifongiques et n'inhibe pas les paramètres de croissance du maïs. Il pourrait être utilisé dans le traitement des semences de maïs. Des recherches pourraient également être effectuées sur ses propriétés herbicides sur certaines légumineuses invasives proches du niébé.

Mots clés: Isoberlinia doka, extrait éthanolique, inhibition, champignons, phytotoxicité

\section{Introduction}

Au Burkina Faso, l'agriculture occupe plus de $85 \%$ de la population active. Cependant les aléas climatiques, la mauvaise qualité des sols, les contraintes liées aux pratiques culturales constituent des problèmes qui limitent la production agricole (Monteiro, 2008). Outre ces contraintes, la pression parasitaire constitue l'une des causes principales de baisse des rendements et de la qualité de la production au Burkina Faso (Dabiré, 2004). En effet, dans l'objectif d'assurer une sécurité alimentaire, les productions agricoles tendent à s'intensifier. Cette intensification s'accompagne le plus souvent d'une augmentation de la population des agents pathogènes (El Guilli et al., 2009). Parmi les agents pathogènes rencontrés, les champignons constituent les plus abondants. Les champignons pathogènes appartenant aux genres Fusarium, Curvularia, Macrphomina, Phoma, Colletotrichum sont les plus répandus, transmis par les semences au Burkina Faso (Somda et al., 2006; Zida et al., 2008). Afin d'éviter les risques de transmission et assurer une 
bonne qualité des semences utilisées, un traitement approprié est nécessaire. Malheureusement, selon Caron \& Laverdière (2006), la complexité des maladies d'origine fongique les rendent difficile à combattre. Le moyen de contrôle de ces champignons phytopathogènes est généralement l'utilisation des fongicides de synthèse. Cette pratique occasionne souvent une utilisation abusive de ces produits chimiques entrainant des conséquences néfastes sur 1'environnement (El Guilli., 2009). Parmi les potentielles alternatives à ces produits chimiques, les biofongicides à base de plantes peuvent constituer des moyens de lutte contre ces champignons (Jacques, 2013). Ainsi, les travaux de plusieurs auteurs ont mis en évidence des propriétés antifongiques des extraits de certaines plantes locales (Bonzi., 2007 ; Somda et al., 2007 ; Bertrand \& Andreu, 2013 ; Kanoun et al., 2014 ; Amine et al., 2017). La présente étude a été initiée dans le but d'évaluer l'efficacité antifongique de l'extrait éthanolique des écorces de Isoberlinia doka sur huit champignons phytopathogènes transmis par les semences.

Isoberlinia doka est une espèces de la famille des Caesalpiniaceae rencontrée dans la région soudanienne en Afrique tropicale, allant du Sénégal (Berhaut, 1971) au lac Tchad (Pias, 1970). C'est un grand arbre à feuilles paripennées, à fleurs blanches avec un large pétale au centre des quatre autres et à fruits s'ouvrant en spirale (Malgras, 1992). Cette plante est localement abondante dans certaines stations de la région soudanienne car elle est l'espèce dominante de certains groupements végétaux (Ouoba, 2006). Sur le plan thérapeutique elle est utilisée en médecine traditionnelle dans le traitement de plusieurs maladies telles que la toux (Kubmarawa al., 2007), les hépatites et les vers intestinaux (Malgras, 1992). L'espèce a déjà montré dans de nombreuses études (Kubmarawa et $a l ., 2007$ ), une activité antifongique contre des champignons opportunistes responsables de maladies infectieuses. Cependant, il existe peu de littérature sur l'activité antifongique sur les agents phytopathogènes de façon générale, et en particulier les champignons transmis par les semences.

\section{Matériel et méthodes:}

\section{Matériel}

\section{Matériel végétal}

Le matériel végétal (Photo1) est constitué d'écorces de tronc de Isoberlinia doka récoltées en mars 2017 dans la forêt classée de Dindêresso à 15 kilomètres à l'Ouest de la ville de Bobo-Dioulasso. L'échantillonnage a été fait sur trois individus de Isobernia doka choisis de manière aléatoire. 


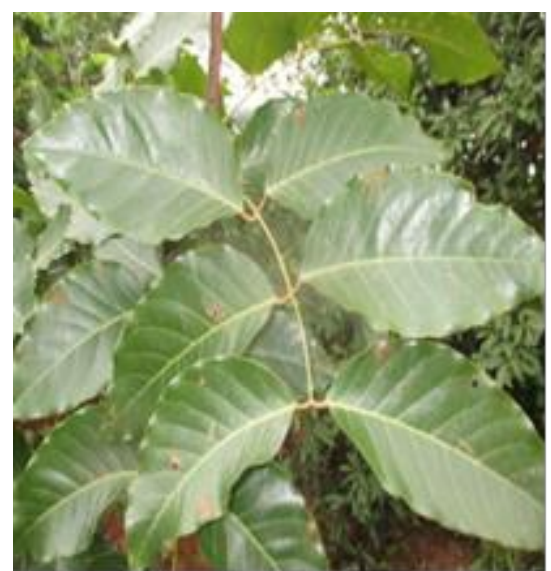

Photo 1 : Isoberlinia doka.

\section{Semences}

Les semences de maïs (Zea mays L.) et niébé (Vigna unguiculata (L.) Walp) ont été fournies par une entreprise spécialisée dans la production et de commercialisation des semences au Burkina Faso.

\section{Matériel fongique}

Le matériel fongique est constitué de huit isolats de champignons phytopathogènes offerts par la clinique des plantes de l'Université Nazi BONI (Tableau I).

Tableau I: Champignons phytopathogènes utilisés

\begin{tabular}{ll}
\hline Champignons phytopathogène & \multicolumn{1}{c}{ Origine } \\
\hline Alternaria alternata (Fr) Keissler & Tomate (Solanum lycopersicum L.) \\
Colletotricum dematium (Pers. Ex Fr) Grove & Niébé (Vigna unguiculata (L.) Walp) \\
Colletotrichum graminicola (Ces) Wilson & Sorgho (Sorghum bicolor (L.) Moench) \\
Curvularia lunata (Wakker) Boedijn & Maïs (Zea mays L.) \\
Fusarium moniliform Sheldom & Sorgho (Sorghum bicolor (L.) Moench) \\
Fusarium oxysporum F.sp. Radicis- & Tomate (Solanum lycopersicum L.) \\
Lycopersici (Sheld) & Maïs (Zea mays L.) \\
Fusarium verticillioides (Sacc) Nirenberg & niébé (Vigna unguiculata (L.) Walp) \\
Macrophomina phaseolina (Tassi) Goid &
\end{tabular}

\section{Methodes}

\section{Préparation de l'extrait éthanolique}

Les écorces prélevées ont été découpées en petits morceaux de 2 à 5 $\mathrm{cm}$ de longueur, puis séchées à la température ambiante du laboratoire et à l'ombre pendant trois semaines. La poudre des écorces a été obtenu à l'aide d'un broyeur (GM-200 Resch). Cent grammes de poudre ont été macéré dans $300 \mathrm{ml} \mathrm{d}$ 'un mélange d'éthanol-eau distillée (70:30 v/v) dans un erlenmeyer 
de 500ml. L'éthanol utilisé est de grade analytique (Carlo Erba) et l'eau distillée est obtenue avec un distillateur (GFL ${ }^{\circledR}$, Typ : 2004). Le mélange obtenu a été soumis à une agitation pendant quarante-huit heures. L'homogénat est filtré successivement deux fois sur du coton hydrophile (labell@) puis sur du papier Wattman. Le filtrât obtenu a subit une évaporation rotative (BUCHI ROTAVAPOR R-200). L'extrait sec obtenu été conservés à $4^{\circ} \mathrm{C}$ avant utilisation. L'opération a été répétée trois fois et le rendement moyen d'extraction a été calculé selon la formule ci-dessous (Ouattara et $a l$, 2011; Kanoun et al., 2014):

$$
R=\left(\frac{m}{M}\right) * 100
$$

$\mathrm{R}$ : le rendement (en pourcentage de l'extraction); $\mathrm{m}$ : la masse de l'extrait brut obtenu après extraction (en gramme) et $\mathrm{M}$ : la masse de la poudre d'écorce (en gramme).

\section{Tests biologiques}

\section{Préparation des milieux de cultures, ensemencement et incubation}

Une solution mère a été préparée en introduisant $1 \mathrm{~g}$ d'extrait dans $10 \mathrm{~mL}$ d'eau distillée, soit une concentration massique de $100 \mathrm{mg} / \mathrm{mL}$. Cette solution a été utilisée pour la préparation du milieu de culture Potato Dextrose Agar (PDA) (Liofilchem ${ }^{\circledR}$, Italy ) à $1 \mathrm{mg} / \mathrm{mL}$. $1 \mathrm{~mL}$ de la solution mère a été ajouté à $99 \mathrm{~mL}$ d'eau contenant 4,2g de PDA. Tous les champignons ont été testés à la concentration de $1 \mathrm{mg} / \mathrm{ml}$. Les champignons les plus sensibles à cette concentration ont été testés aux doses de 2,$5 ; 5$ et $10 \mathrm{mg} / \mathrm{ml}$. Ces dernières concentrations ont été obtenues en ajoutant respectivement 2,$5 ; 5$ et $10 \mathrm{~mL}$ de la solution mère à 97,$5 ; 95$ et $90 \mathrm{~mL}$ d'eau distillée contenant 4,2 $\mathrm{g}$ de PDA.

Le milieu de culture servant de "témoin eau' a été préparé en mélangeant 4,2g de PDA (Liofilchem ${ }^{\circledR}$, Italy ) à $100 \mathrm{~mL}$ d'eau distillée. Ces milieux de cultures ainsi préparés ont été stérilisés à l'autoclave $(\mathrm{PbI})$ à $120^{\circ} \mathrm{C}$ pendant $30 \mathrm{mn}$. Après refroidissement à $60^{\circ} \mathrm{C}$, ces milieux ont été répartis dans des boîtes de pétri de $90 \mathrm{~mm}$ de diamtètre (Aptaca Italy).

Le milieu témoin à base de fongicide a été préparé en mélangeant $4,2 \mathrm{~g}$ de PDA à $100 \mathrm{~mL}$ d'eau distillée puis stérilisé dans les mêmes conditions que précédemment. Après refroidissement à $60^{\circ} \mathrm{C}, 0,4 \mathrm{~g}$ de Calthio $\mathrm{C}(25 \%$ chloroform-ethyl $+25 \%$ Thirame, WS) a été ajouté au mélange eau-PDA, homogénéisé puis réparti dans des boîtes de pétri.

Les boîtes de pétri contenant les milieux de culture ont été ensemencées par des explants de mycélium âgé de 10 jours dans des conditions aseptiques sous une hotte à flux laminaire (Napflow 12STD GV2EFR). Les boîtes de pétri ont été scellée avec du parafilm (Parafilm®, Neemah, Wi54956), puis incubées à $22-23^{\circ} \mathrm{C}$ pendant 12 heures sous la 
lumière proche Ultra-Violet (PhilipsTLD 36W / 08) alterné avec 12 heures d'obscurité (Mathur et Kongsdal, 2003).

\section{Evaluation de la croissance radiale mycélienne}

L'évaluation de la croissance radiale a été faite quatre et sept jours après incubation (JAI). Deux droites perpendiculaires passant par le centre de l'explant ont été tracées sur le couvercle de la boîte de pétri et servant à mesurer le diamètre du mycélium. La moyenne arithmétique des mesures faites sur les deux droites perpendiculaires a constitué le diamètre moyen de croissance mycélienne. Le pourcentage moyen d'inhibition de la croissance mycélienne a été calculé selon la formule suivante :

$$
I=\frac{D t-D e}{D t} * 100
$$

I : pourcentage moyen d'inhibition de la croissance mycélienne ; Dt : diamètre moyen d'inhibition du témoin eau et De : diamètre moyen d'inhibition de l'extrait ou du fongicide.

\section{Evaluation de la phytotoxicité in vivo}

La phytotoxicité a été évaluée sur le maïs et le niébé selon trois traitements en raison de 100 graines par traitement. Les différents traitements sont : Le témoin eau, le témoin fongicide à raison de $20 \mathrm{~g}$ de Calthio $\mathrm{C} / 5 \mathrm{~kg}$ de semences et 1'extrait éthanolique à $10 \mathrm{mg} / \mathrm{ml}$. Les graines traitées ont été incubés à la température ambiante pendant 24 heures et semées dans des pots contenant du sable fin stérile en quatre répétitions à raison de 20 graines par répétition. Après semis, les pots ont été disposés sous un tunnel à la température ambiante(Mathur et Kongsdal, 2003). L'évaluation de la phytotoxicité a consisté à compter le nombre de plantules émergés au quatrième et septième jour après semis pour le maïs et au cinquième et au huitième jour pour le niébé. La hauteur des tiges, la longueur des racines et la masse de dix plantules de maïs et de niébé prises au hasard dans chaque répétition ont été mesurées pour la détermination de la biomasse produite 10 jours après semis.

\section{Analyse des données}

Le logiciel XLSTAT 2007 a servi à analyser les données. La croissance radiale moyenne de chaque champignon ainsi que les moyennes des taux d'émergence, de la hauteur des tiges, de la longueur des racines et de la masse des plantules pour chaque traitement ont été comparés au seuil de 5\% selon le test de comparaison multiple de Student-Newman-Keuls. 


\section{Résultats et discussion \\ Résultats}

\section{Rendement de l'extraction}

Après extraction, nous avons obtenu un rendement en extrait brut sec de $5,27 \%$. Cet extrait a été conservé à l'abri de $1^{\prime}$ humidité à $4^{\circ} \mathrm{C}$ avant son utilisation.

\section{Croissance mycélienne à $\mathbf{4}$ jours après incubation}

Les résultats de la croissance mycélienne au quatrième jour sont consignés dans le tableau II. A cette date, nous avons noté que les champignons les plus sensibles à l'extrait sont $C$. Dematium, $F$. verticilloides et $C$. Lunata. avec des pourcentages d'inhibition respectifs de 18,93, 17,19 et $10,42 \%$.

Tableau II : Croissance mycélienne à quatre jours après incubation

\begin{tabular}{lcccccccc}
\hline \multirow{2}{*}{ Traitements } & \multicolumn{7}{c}{ Croissance mycélienne (cm) } \\
\cline { 2 - 9 } & C.dem & C.lun & F.ver & F.mon & F.oxy & M.pha & C.gra & A.alt \\
\hline TF & $0,50^{\mathrm{a}}$ & $0,50^{\mathrm{a}}$ & $0,50^{\mathrm{a}}$ & $0,50^{\mathrm{a}}$ & $0,50^{\mathrm{a}}$ & $0,50^{\mathrm{a}}$ & $0,50^{\mathrm{a}}$ & $0,50^{\mathrm{a}}$ \\
$\mathrm{TE}$ & $4.28^{\mathrm{c}}$ & $4,80^{\mathrm{c}}$ & $4,77^{\mathrm{c}}$ & $4,13^{\mathrm{b}}$ & $3,37^{\mathrm{b}}$ & $7,80^{\mathrm{b}}$ & $4,35^{\mathrm{b}}$ & $1,62^{\mathrm{b}}$ \\
$\mathrm{EE}(1 \mathrm{mg} / \mathrm{mL})$ & $3.47^{\mathrm{b}}$ & $4,30^{\mathrm{b}}$ & $3,95^{\mathrm{b}}$ & $3,98^{\mathrm{b}}$ & $3,28^{\mathrm{b}}$ & $7,32^{\mathrm{b}}$ & $4,17^{\mathrm{b}}$ & $2,00^{\mathrm{c}}$ \\
\hline & $<0,000$ & & $<0,000$ & $<0,000$ & $<0,000$ & 0,000 & 0,000 & $<0,000$ \\
$P$ & 1 & $<0,0001$ & 1 & 1 & 1 & 1 & 1 & 1 \\
\hline $\mathrm{I}(\%)$ & 18,93 & 10,42 & 17,19 & 3,62 & 2,67 & 6,15 & 4,14 & - \\
\hline
\end{tabular}

Dans une même colonne, les moyennes affectées de la même lettre alphabétique ne sont pas significativement différentes au seuil de $5 \%$, suivant le test de classification multiple de StudentNewman-Keuls. C. dem : C. dematium; C. lun : C. lunata; F. vert : F. verticilloides; F. mon: F. moniliforme ;F. oxy: F. oxysporum; M.pha: M. phaseolina; C. gra: C. graminicola; A.alt : A. alternata. TF: témoin fongicide; TE: témoin eau; EE extrait éthanolique; P: probabilité et I: pourcentage d'inhibition.

\section{Croissance mycélienne à sept jours après incubation}

Les résultats de la croissance mycélienne au septième jour après incubation sont consignés dans le tableau III. Au septième jour les champignons les plus sensibles à l'extrait reste toujours les mêmes qu'au quatrième jour après incubation. A cette date, nous avons noté des pourcentages d'inhibition de 32,$80 ; 12,66$ et 12,33 respectivement pour $C$. Dematium, $C$. Lunata et $F$. verticilloides. 
Tableau III : Croissance mycélienne à 7 jours après incubation

\begin{tabular}{lcccccccc}
\hline & \multicolumn{7}{c}{ Croissance mycélienne (cm) } \\
\cline { 2 - 9 } Traitements & C.dem & C.lun & F.ver & F.mon & F.oxy & M.pha & C.gra & A.alt \\
\hline TF & $0,50^{\mathrm{a}}$ & $0,50^{\mathrm{a}}$ & $0,50^{\mathrm{a}}$ & $0,50^{\mathrm{a}}$ & $0,50^{\mathrm{a}}$ & $0,50^{\mathrm{a}}$ & $0,50^{\mathrm{a}}$ & $0,50^{\mathrm{a}}$ \\
$\mathrm{TE}$ & $6,28^{\mathrm{c}}$ & $7,82^{\mathrm{c}}$ & $7,87^{\mathrm{c}}$ & $6,55^{\mathrm{b}}$ & $5,72^{\mathrm{b}}$ & $8,58^{\mathrm{b}}$ & $6,95^{\mathrm{b}}$ & $2,13^{\mathrm{b}}$ \\
$\mathrm{EE}$ & $4.22^{\mathrm{b}}$ & $6,83^{\mathrm{b}}$ & $6,9^{\mathrm{b}}$ & $6,32^{\mathrm{b}}$ & $5,57^{\mathrm{b}}$ & $8,53^{\mathrm{b}}$ & $7,58^{\mathrm{c}}$ & $2,42^{\mathrm{c}}$ \\
\hline$P$ & $<0,0001$ & $<0,0001$ & $<0,0001$ & $<0,0001$ & $<0,0001$ & $<0,0001$ & $<0,0001$ & $<0,0001$ \\
\hline $\mathrm{I}$ & 32,80 & 12,66 & 12,33 & 3,51 & 2,62 & 0,58 & - & - \\
\hline
\end{tabular}

Dans une même colonne, les moyennes affectées de la même lettre alphabétique ne sont pas significativement différentes au seuil de $5 \%$, suivant le test de classification multiple de StudentNewman-Keuls. C. dem : C. dematium; C. lun : C. lunata ; F. vert : F. verticilloides; F. mon : F. moniliforme;F. oxy: F. oxysporum; M.pha: M. phaseolina; C. gra: C. graminicola; A.alt: A. alternata. TF: témoin fongicide; TE: témoin eau; EE extrait éthanolique; P: probabilité et I: pourcentage d'inhibition.

\section{Effet de la variation de la concentration d'extrait sur la croissance mycélienne}

Les pourcentages d'inhibition de la croissance des trois champignons les plus sensibles ont varié avec l'augmentation de la concentration de l'extrait éthanolique (tableau IV). Au quatrième jour après incubation, à la concentration de $10 \mathrm{mg} / \mathrm{mL}$, nous avons noté respectivement des pourcentages d'inhibition de 52,$35 ; 43,12$ et $32,63 \%$ sur $C$. lunata ( Photo 2) , F. verticilloides et $C$. dematium. Au septième jour après incubation à la même concentration de $10 \mathrm{mg} / \mathrm{mL}$, nous avons noté les mêmes tendances à l'inhibition avec cependant une inhibition plus renforcée sur F.verticilloides.

Tableau IV: Pourcentage d'inhibition de $C$. dematium, $C$. lunata et $F$. verticilloides quatre et sept jours après incubation

\begin{tabular}{ccccccc}
\hline \multirow{2}{*}{$\begin{array}{c}\text { Concentration en } \\
\text { extrait }(\mathbf{m g} / \mathbf{m L})\end{array}$} & \multicolumn{5}{c}{ C. dematium } & \multicolumn{2}{c}{ C. lunata } & \multicolumn{2}{c}{$\boldsymbol{F}$. verticilloides } \\
\cline { 2 - 7 } & $4 \mathrm{JAI}$ & $7 \mathrm{JAI}$ & $4 \mathrm{JAI}$ & $7 \mathrm{JAI}$ & $4 \mathrm{JAI}$ & $7 \mathrm{JAI}$ \\
\hline & $21,30^{\mathrm{a}}$ & $19,51^{\mathrm{a}}$ & $40,12^{\mathrm{a}}$ & $18,30^{\mathrm{a}}$ & $12,98^{\mathrm{a}}$ & $32,35^{\mathrm{a}}$ \\
2,5 & $26,37^{\mathrm{a}}$ & $20,31^{\mathrm{a}}$ & $47,14^{\mathrm{b}}$ & $25,93^{\mathrm{b}}$ & $31,39^{\mathrm{b}}$ & $44,30^{\mathrm{b}}$ \\
5 & $32,63^{\mathrm{a}}$ & $30,48^{\mathrm{b}}$ & $52,35^{\mathrm{b}}$ & $35,51^{\mathrm{c}}$ & $43,12^{\mathrm{b}}$ & $50,70^{\mathrm{b}}$ \\
\hline 10 & 0,12 & 0,0001 & 0,006 & $<0.0001$ & 0,004 & 0,005 \\
\hline Probabilité & & &
\end{tabular}

Dans une même colonne, les moyennes affectées de la même lettre alphabétique ne sont pas significativement différentes au seuil de $5 \%$, suivant le test de classification multiple de StudentNewman-Keuls. JAI : Jour après incubation 


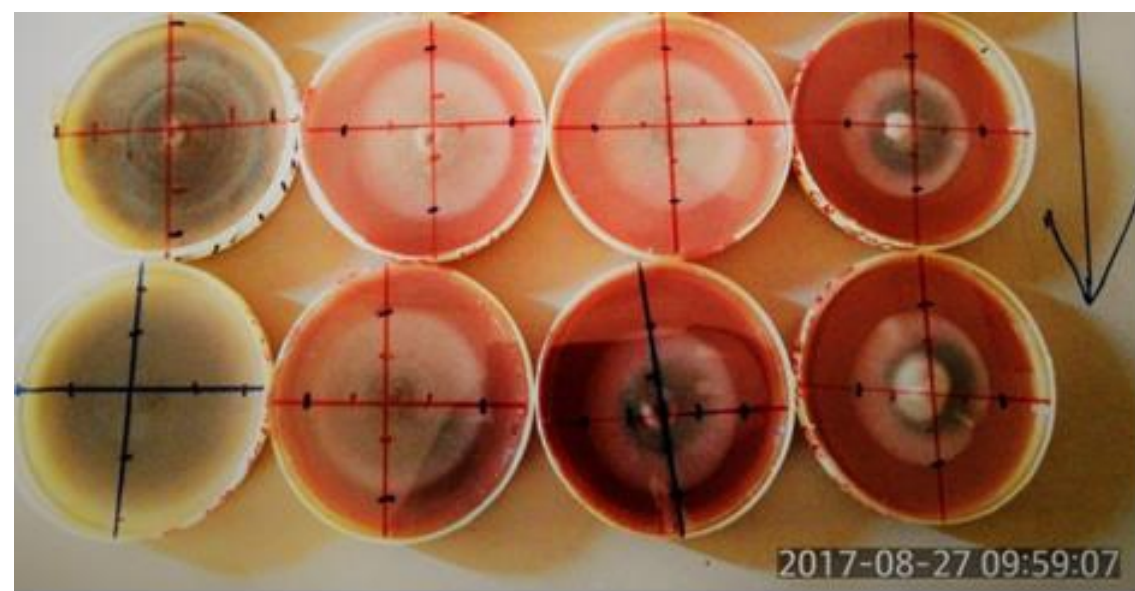

Témoin eau

Extrait

$(2,5 \mathrm{mg} / \mathrm{mL})$

Extrait

Extrait

$(5 \mathrm{mg} / \mathrm{mL})$

$(10 \mathrm{mg} / \mathrm{mL})$

Photo 2 : Croissance mycélienne de $C$. lunata, champignon le plus sensible au quatrième jour après incubation

Effet des traitements sur la germination des grains de maïs et de niébé

La figure 2 montre l'effet des traitements sur la germination du maïs et du niébé. Au quatrième jour après semis, les taux de germination varient de $93 \%$ pour le témoin eau à $96 \%$ pour le témoin fongicide. Au septième jour après semis ces taux varient de 96 à $98 \%$. Comparativement aux deux témoins, l'extrait n'a pas d'effet d'inhibition significatif au seuil de $5 \%$ sur la germination du maïs. Au cinquième jour après semis les taux de germination du niébé ont été de $26 \%$ pour l'extrait éthanolique, $88 \%$ pour le fongicide et de $99 \%$ pour le témoin eau. Au huitième jour après semis les taux de germination ont été de $66 \%$ pour l'extrait éthanolique, $97 \%$ pour le fongicide et de $99 \%$ pour le témoin eau. Il n'y a pas de différence significative entre les taux de germination au niveau du fongicide et du témoin eau. Par contre, l'extrait inhibe la germination du niébé au cinquième et au huitième jour après semis. 


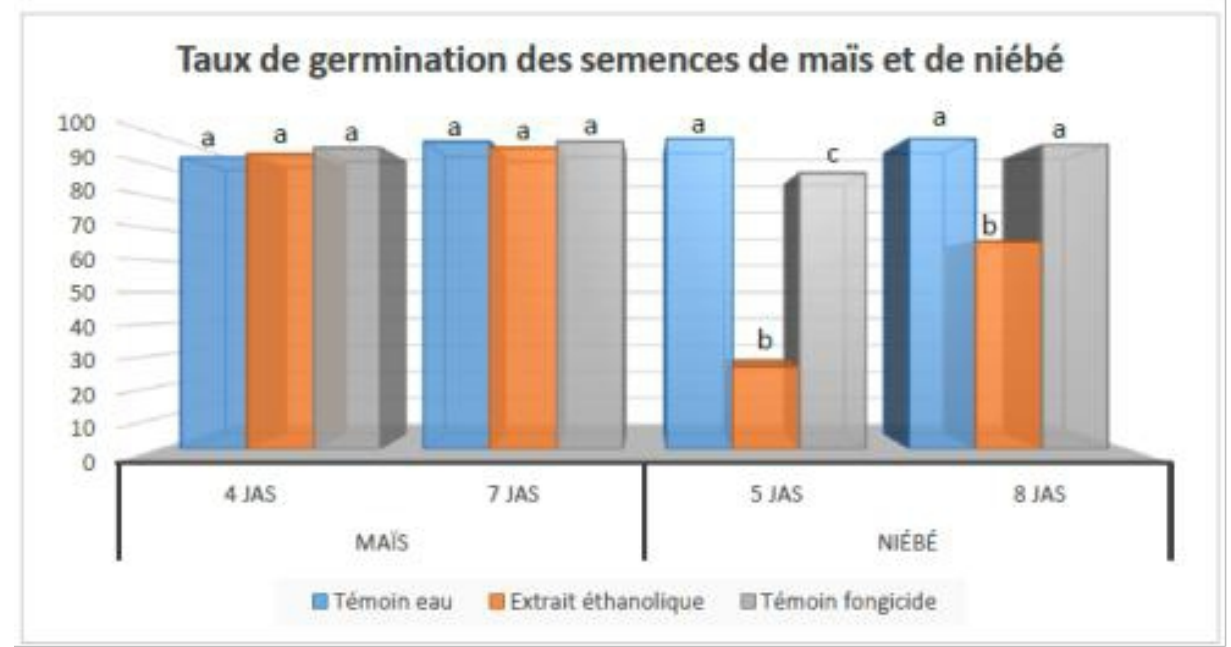

Figure 2: Effet traitements sur la germination des grains de maïs et de niébé JAS : jour après semis. Les colonnes affectées de la même lettre alphabétique ne sont pas significativement différentes au seuil de $5 \%$, suivant le test de classification multiple de Student-Newman-Keuls.

\section{Effet de l'extrait éthanolique sur la biomasse du maïs et du niébé}

Le tableau VII présente la longueur des racines, la hauteur des tiges et la masse des plantules de maïs dix jours après semis. L'analyse de variance ne montre pas de différences significatives entre les traitements $(p>0,05)$ (Photo 3 A). Par contre pour ce qui est du niébé, l'analyse statistique de la hauteur des tiges et de la masse des plantules montre des différences hautement significatives entre les traitements à dix jours après semis (tableau VII). Il n’y a pas de différence significative entre la longueur des racines au niveau des différents traitements contrairement à la longueur des tiges et à la masse des plantules. En effet, comparativement aux deux témoins, les plantules issues des semences traitées avec l'extrait éthanolique sont de faible masse et ont des tiges moins longues. L'extrait a un effet inhibiteur sur la croissance des plantules du niébé (Photo $3 \mathrm{~B}$ ).

Tableau I : Effet de l'extrait éthanolique sur la biomasse du maïs et de niébé à 10 jours après semis

\begin{tabular}{lcccccc}
\hline & \multicolumn{3}{c}{ Maïs } & & Niébé & \\
\cline { 2 - 7 } Traitements & $\begin{array}{c}\text { Longueur } \\
\text { des racines } \\
(\mathrm{cm})\end{array}$ & $\begin{array}{c}\text { Hauteur } \\
\text { des tiges } \\
(\mathrm{cm})\end{array}$ & $\begin{array}{c}\text { Masse des } \\
\text { plantules } \\
(\mathrm{g})\end{array}$ & $\begin{array}{c}\text { Longueur } \\
\text { des racines } \\
(\mathrm{cm})\end{array}$ & $\begin{array}{c}\text { Hauteur } \\
\text { des tiges } \\
(\mathrm{cm})\end{array}$ & $\begin{array}{c}\text { Masse } \\
\text { des } \\
\text { plantules }(\mathrm{g})\end{array}$ \\
\hline TA & $31,7^{\mathrm{a}}$ & $15,99^{\mathrm{a}}$ & $19,72^{\mathrm{a}}$ & $15,06^{\mathrm{a}}$ & $20,2^{\mathrm{a}}$ & $20,02^{\mathrm{a}}$ \\
$\mathrm{F}$ & $31,2^{\mathrm{a}}$ & $16^{\mathrm{a}}$ & $19,97^{\mathrm{a}}$ & $14,77^{\mathrm{a}}$ & $16,42^{\mathrm{b}}$ & $18,66^{\mathrm{b}}$ \\
$\mathrm{EE}$ & $30,63^{\mathrm{a}}$ & $15,83^{\mathrm{a}}$ & $19,30^{\mathrm{a}}$ & $13,43^{\mathrm{a}}$ & $14,39^{\mathrm{c}}$ & $15,83^{\mathrm{c}}$ \\
$\mathrm{P}$ & 0,52 & 0,94 & 0,79 & 0,35 & $<0,0001$ & $<0,0001$
\end{tabular}

Dans une même colonne, les moyennes affectées de la même lettre alphabétique ne sont pas significativement différentes au seuil de $5 \%$, suivant le test de classification multiple de StudentNewman-Keuls. 


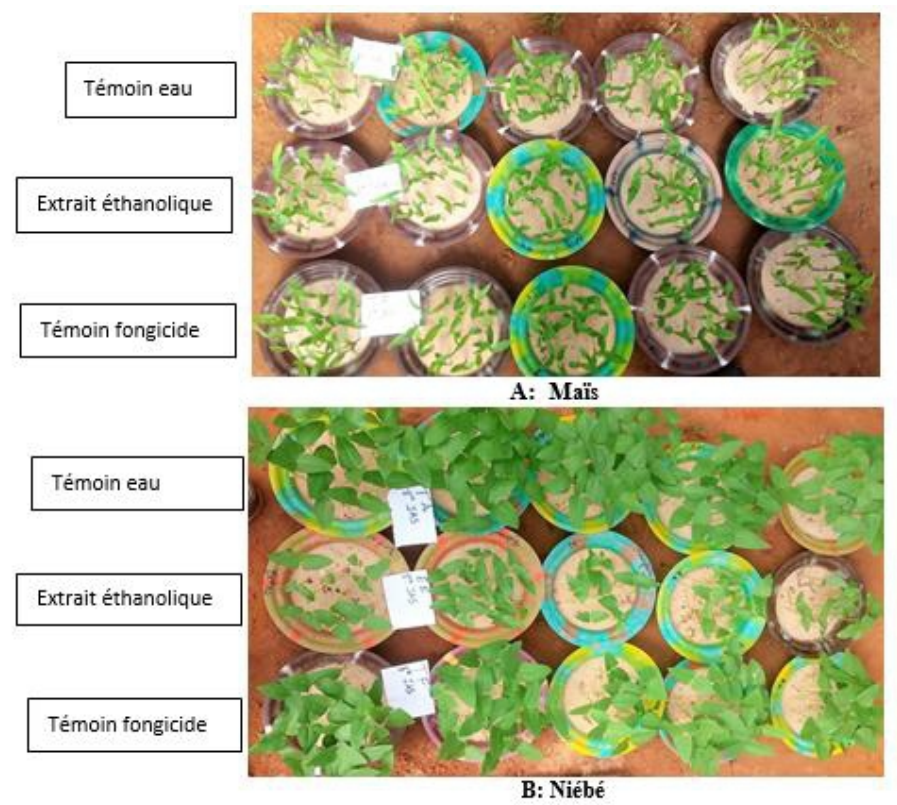

Photo 3 : Plantules de maïs (A) et de niébé (B) huit jours après semis

\section{Discussion}

Cette étude avait pour objectif d'évaluer l'activité antifongique de l'extrait éthanolique des écorces de I. doka sur huit champignons transmis par les semences.

Après extraction par macération hydroalcoolique, un rendement de 5\% en extrait sec a été obtenu. Plusieurs facteurs peuvent influencer le rendement en extraits. La quantité de métabolites secondaires et leur nature, dépend des conditions physiologiques et écologiques de la plante et de la période de récolte (Tsopmbeng et al., 2014). Les facteurs cités ci-dessus, peuvent conduire à une réduction très significative de certaines molécules (Belmokhtar, 2015).

L'évolution du développement mycélien en fonction du temps montre une efficacité de l'extrait à des degrés différents entre les champignons. Les résultats de l'évaluation de la croissance radiale ont montré que $C$. dematium, $C$. lunata et $F$. verticilloides sont les plus sensibles à l'extrait à $10 \mathrm{mg} / \mathrm{ml}$. A cette concentration, on a noté une inhibition maximale de C. lunata $(52,35 \%)$ au quatrième jour après incubation alors qu'au septième jour après incubation, l'inhibition maximale a été observée sur $F$. verticilloides $(50,70 \%)$. Plusieurs travaux ont montré que les extraits hydroalcooliques végétaux étaient doté d'activité antifongique (Kubmarawa et al., 2007 ; Yaye et al., 2011). Cette propriété antifongique pourrait être due aux composés tels que les flavonoïdes (Ilboudo et al., 2016; Afolayan et al., 1997), les alcaloïdes (Mezouar et al., 2014) les saponosides (Saniewska et al., 2006) et les terpénoïdes (Belaiche et 
al., 2000). Ces molécules seraient synthétisées par les plantes, afin de résister aux parasites tels que les insectes et les champignons. Elles seraient à la base des propriétés fongicides (Orsot et al., 2015). Ces molécules actives peuvent agir individuellement ou en synergie.

Quant aux tests de phytotoxicité, les résultats ont montré qu'à $10 \mathrm{mg} / \mathrm{ml}$, l'extrait n'a pas d'effet significatif sur la germination du maïs comparativement au témoin eau et au témoin fongicide. Le plus faible taux de germination des semences de maïs traitée à l'extrait éthanolique était de $94 \%$ au quatrième jour après semis et de $96 \%$ au septième jour après semis. La mesure des paramètres de croissance (hauteur des tiges, longueur des racines et masse des plantules) n'a pas montré de différence significative avec les témoins.

Cependant, l'extrait éthanolique a inhibé la germination des semences et la croissance des plantules du niébé. L'extrait a une phytotoxicité sur les semences du niébé à $10 \mathrm{mg} / \mathrm{ml}$ entrainant un faible taux de germination des semences et une réduction de la biomasse. Ce résultat pourrait s'expliquer par la concentration forte, ou la présence dans l'extrait, de constituants qui auraient empêché et retardé la germination des grains du niébé. En effet, en plus des propriétés antifongiques, les composés présents dans les extraits hydroalcooliques peuvent être phytotoxiques sur certaines espèces végétales (Benmeddour et Fenni, 2018 ; Mecina et al., 2016 ; Bertrand et Andreu, 2013). Ces résultats confirment que l'extrait éthanolique de $I$. doka pourrait être utilisé en traitement des semences contre les champignons du maïs qui est une plante vivrière de première importance en Afrique de l'Ouest.

\section{Conclusion}

Au terme de notre étude, les résultats obtenus montrent que l'extrait éthanolique a réduit à des degrés variables le développement des champignons téstés. L'extrait éthanolique a montré une efficacité intéressante sur trois champignons : $C$. dematium, $C$. lunata et $F$. verticilloides. Parmi ces champignons, $C$. lunata a été le plus sensible avec un taux d'inhibition de sa croissance mycélienne de 52,35\% après quatre jours d'incubation à $10 \mathrm{mg} / \mathrm{ml}$. A sept jours d'incubation, l'extrait a été plus efficace dans la réduction de la croissance mycélienne de $F$. verticilloides avec un taux d'inhibition de plus de $50 \%$ à la même concentration. Pour les tests de phytotoxicité, l'extrait n'a pas d'effet phytotoxique sur la germination et la croissance des plantules de maïs. Par contre, il a entrainé une réduction significative de la germination des graines et de la croissance des plantules du niébé. L'extrait éthanolique des écorces de I. doka possède des propriétés antifongiques et non toxique sur le maïs. Il pourrait être utilisé en traitement des semences de maïs. De plus, l'extrait ayant montré une phytotoxicité sur le niébé, on pourrait s'intéresser à ses propriétés herbicides contre certaines légumineuses envahissantes. 


\section{Remerciements}

Nous remercions toute l'équipe des laboratoires de Biologie végétale et de Biochimie de l'Université Nazi BONI pour leurs contributions techniques pendant l'identification des plantes et l'extraction. Nos remerciements vont également à la Clinique des Plantes de l'Université Nazi BONI en particulier au Pr. SOMDA Irénée pour nous avoir fourni le plateau technique pour les tests antifongique et de phytoxicité.

\section{References:}

1. Afolayan A.J, Meyer J.J. (1997). The antimicrobial activity of 3,5,7trihydroxyflavone isolated from the shoots of Helichrysum aureonitens. J Ethnopharmacol, 57 (3):177-81.

2. Amine D., Mohamed B., Zoubida H., Jamal I., Laila N. (2017). Activité antifongique des extraits aqueux de Calendula officinalis L, Urginea maritima (L.) Baker et Chenopodium ambrosioides L. European Scientific Journal, 13 (24): 483-497.

3. Belaiche T., El Halouat A., Tantaoui Elaraki A. (2000). Etude de l'influence des terpènes sur la sporulation d'Aspergillus. AGRIS, 116 (6): $27-29$.

4. Belmokhtar Z. (2015). Identification et caractérisation des molécules du métabolisme secondaire de Retama monosperma. L Boiss, intérêt pharmaceutique. Doctorat en Sciences de l'Université des Sciences et de la Technologie d'Oran Mohamed Boudiaf.

5. Benmeddour T., Fenni M. (2018). Phytotoxicité des extraits de trois espèces végétales sur le blé dur et sur Kochia scoparia: adventice envahissante des périmètres gricoles dans la Wilaya de Biskra. Courrier du Savoir, 25:173-178.

6. Berhaut J. (1971). Flore illustrée du Sénégal. Ministère du Développement Rural et de 1'Hydraulique, Direction des Eaux et Forêts, Dakar. Tome 1, 626p.

7. Bertrand C., \& Andreu V. (2013). Caractéristiques et intérêts des préparations à base de plantes. Journées Substances Naturelles en Protection des Cultures: Réglementation, expérimentation, usages. Colloque Intrants Naturels ITAB/GRAB - 9\&10 Paris.

8. Bonzi S. (2007). Efficacité des extraits aqueux de quatre plantes dans la lutte contre les champignons transmis par les semences de sorgho (Sorghum bicolor (L.) Moench): Cas particulier de Colletotricum graminicola (Ces.)Wilson et Phoma soghina (Sacc.)Boerema, Dorenbosch et Van Kesteren. Diplôme d'Etudes Approfondies en Gestion intégrée des Ressources Naturelles. Université Polytechnique de Bobo-Dioulasso, Bobo-Dioulasso, Burkina Faso. 
9. Caron J. \& Laverdière L. (2006). Recherche et développement de biopesticides et pesticides naturels à faible toxicité pour les organismes non ciblés et respectueux de l'environnement. Rapport final- volet Phytopathologie. Université Laval, Québec.

10. Dabiré G.T. (2004). Etude de l'efficacité d'extrait végétaux contre les agents pathogènes fongiques transmis par les semences de mil et de sorgho. Mémoire d'Ingénieur de développement rural. Université polytechnique de Bobo-Dioulasso, Bobo-Dioulasso, Burkina Faso.

11. El Guilli M., Achbani E., Fahad K., Jijakli H.(2009). Biopesticides : Alternatives à la lutte chimique? Symposium international AGDUMED. Rabbat, Maroc: 266-280.

12. Ilboudo O., Bonzi S., Tapsoba I., Somda I., Bonzi - Coulibaly Y.L. ,2016 Activité antifongique in vitro des flavonoïdes diglycosides de Mentha piperita et de leur dérivé oxime contre deux champignons céréaliers. Comptes Rendus Chimie, 19 (7): 857-862.

13. Jacques S. (2013). Produits naturels de protection des plantes et les opportunités de développement. Journées Substances Naturelles en Protection des Cultures: Réglementation, expérimentation, usages. Colloque Intrants Naturels ITAB/GRAB - 9\&10, Paris.

14. Kanoun K., Abbouni B., Bénine M.L., Benmahdi F.Z., Marouf B. (2014). Étude de l'efficacité de l'extrait éthanolique d'écorces de Punica granatum Linn. sur deux souches phytopathogènes : Ascocyhta rabiei (pass.) labr. et Fusarium oxysporum f. sp. radicis-lycopersici. European Scientific Journal, 10 (12): 301-315.

15. Kubmarawa D., Ajoku G.A., Enwerem N.M, Okorie D.A. (2007). Preliminary phytochemical and antimicrobial screening of 50 medicinal plants from Nigeria. African Journal of Biotechnology, 6 (14):1690-1696.

16. Malgras D. (1992). Arbres et Arbustes guérisseurs des savanes Maliennes. Editions karthala Acct, 478p.

17. Mathur S.D., Kongsdal O. (2003). Common Laboratory Seed Health Testing Methods for detecting fungi. First edition, Kandrups Bogtrykkeri edition, 436p.

18. Mecina G.F., Santos V.H.M., Andrade A.R., Dokkedal A.L., Saldanha L.L., Silva L.P, Silva R.M.G. (2016) Phytotoxicity of Tridax procumbens L. South African Journal of Botany, 102:130-136.

19. Mezouar D., Lahfa F.B., Abdelouahid D.E., Adida H., Rahmoun N.M., Boucherit-Otmani Z. (2014). Activité antimicrobienne d'extraits d'écorce de racines de Berberis vulgaris. Phytothérapie, 12(6): 38085.

20. Monteiro J.F. (2008). Contrôle biologique de la pourriture cendrée (Macrophomina phaseolina) du niébé par l'enrobage des semences 
avec Clonostachys rosea: Impact des conditions de conservation des semences traitées sur la performance du bioagent. Mémoire d'Ingénieur en protection des végétaux, Centre Regional Agrhymet, Niamey, Niger.

21. Orsot B.A.M.B., Soro S., Ouattara D., N'guessan E.K., Zirihi G.N. (2015). Étude ethnobotanique et évaluation in vitro de l'activité antifongique des extraits de feuilles de Mallotus oppositifolius sur deux souches phytopathogènes de Sclerotium rolfsii. European Scientific Journal, 11(36): 418-435.

22. Ouattara L., Koudou J., Zongo C., Barro N., Savadogo A., Bassole I.H.N., Ouattara A.S.,Traore A.S. (2011). Antioxidant and Antibacterial Activities of Three Species of Lannea from Burkina Faso. Journal of Applied Sciences, 11 (1): 157-162.

23. Ouoba P. (2006). Flore et végétation de la forêt classée de Niangoloko, Sud-Ouest du Burkina Faso.Thèse de doctorat, Université de Ouagadougou, Burkina Faso.

24. Pias J. (1970). La végétation du Tchad. Travaux et documents de l'ORSTOM, $\mathrm{N}^{\mathrm{O}}$ 6, Paris, 47p.

25. Saniewska A., Jarecka A., Bialy Z., Jurzyst M. (2006). Antifungal activity of saponins originated from Medicago hybrida against some ornamental plant pathogens. Acta Agrobotanica, 59(2): 5158.

26. Somda I., Leth V., Sérémé P. (2007). Antifungal effect of Cymbopogon citratus, Eucalyptus camaldulensis and Azadirachta indica oil extracts on Sorghum seed-borne fungi. Asian Journal of Plant Sciences, 6:1182-1189

27. Somda I., Leth V., Séréme P. (2006). Seed-borne fungi of food and cash crops grown in Burkina Faso. Etudes et Recherches Sahéliennes, 13: 56-66.

28. Tsopmbeng N.G., Megatche C. J.P., Lienou J.A., Aoudou Y., Djeugap F.J., Fontem D.A. (2014). Évaluation des activités antifongiques des extraits de plantes contre Phytophthora colocasiae, agent causal du mildiou du taro (Colocasia esculenta (L.) Schott). Journal of Applied Biosciences, 81: 7221-7232.

29. Yaye G.Y., Kra M.K.A., Ackah J.A.A.B., Djaman A.J. (2011). Évaluation de l'activité antifongique et essai de purification des principes actifs des extraits de Terminalia mantaly (H. Perrier), une combrétacée, sur la croissance in vitro de Candida albicans. Bulletin de la Société Royale des Sciences de Liège, 80: 953-964.

30. Zida P.E., Sérémé P., Leth V., Sankara P., Somda I., Neya A., 2008. Importance of seed-borne fungi of sorghum and pearl millet in Burkina Faso and their control using plant extracts. Pakistan Journal of Biological. Sciences, 11 (3): 321-331. 